\title{
How Electricity has Handicapped Robots: Reliance on Electricity has Made Robots Into Rollbots
}

Allyn JL*

RIL Robots, LLC, Los Angeles, USA

\section{Introduction}

Los Angeles, CA: The reliance on electricity has put walking robots financially out of reach of consumers. Electricity is the Achilles heel of life-size powered robots. It has handicapped what would otherwise be robots that could benefit humanity.

The Honda Asimo walking robot, as an example of the out-of-reach drawback of electricity-powered robots, cost $\$ 1$ million to build but can only walk for 30 minutes on a battery charge. Moreover, it wouldn't be anybody's first thought who might have a spare million dollars laying around, of spending the money on a Honda Asimo. Especially, when it only walks for 30 minutes.

To put that into perspective, a walk on the Santa Monaca Beach Pier in Los Angeles, takes an hour to walk from the front of the pier, out to the end and back. For all the technology it possesses, the Honda Asimo would not be able to walk back from the end of the pier and given the entertainment that lines the Santa Monica Beach Pier, stopping to view the various performances along the way would cause the Asimo robot to not even make it to the end of the pier. Let alone the trip back. It would need a wheelchair to get back to the car.

There's another example of a highly technological robot having to be sidelined to wheels. When Aldebaran decided to release its "Pepper" emotion-reading robot, during the unveiling to the media, the CEO said there was one reason it was on wheels and not walking. Because walking would have consumed $85 \%$ of its battery.

\section{Rollbots}

Why all this reliance on electricity for robots? A reliance that has turned robots into "rollbots." And rollbots, not having the capacity of climbing steps or stairs, are confined to a single indoor floor. Even outside, assuming they might fall over negotiating access ramps, they are confined to a single level surface.

One company has decided to do things differently. Instead, it has completely eliminated powered walking from the locomotion of its robots. RIL Robots, LLC in Los Angeles [1], has developed a robot that looks like a human, but walks without motors or electricity. And seeing it for the first time, one wonders how a robot walking without motors or electricity is even possible (Figure 1) [2].

\section{RIL Companions}

Its robots, called "RIL Companions," are developed to provide comfort and robot companionship to the shy, the lonely and the elderly. Without electricity, they can also walk up and down single steps and access ramps. The can even negotiate steep ramps of loading docks.

Since they are structured like humans, with a stainless steel skeleton and life-like TPE skin, RIL Companion walking robots can participate in many activities as humans can. Activities that rollbots never could do.

RIL Companions are designed to be walked to a car, get in, and be driven anywhere where they can then get out. They can walk on the beach or in the mall. They can ride on the back of a motorcycle, jet ski, or on the back seat of a tandem bike.

But best of all, they can walk endlessly. Not only can they be walked to the end of the Santa Monica Beach Pier and back. After doing so, they can then walk on Santa Monica Promenade, sit and sip on a water at Starbucks, go watch a movie, and then walk back to where the car is parked. All without a single drop of electricity to walk and while RIL Companions do not offer the highly advanced features of an advance robot such as Honda's Asimo, they offer the one thing that matters most. Affordability. The RIL Companion base price startts around just less than a human's annual health insurance bill and with it's artificial intelligence, it can hold a conversation for an hour or more. As good as, if not better than, with a human.

Because of the affordability, the company also plans to offer its robots as robot fashion models, a tremendous upgrade from fiberglass

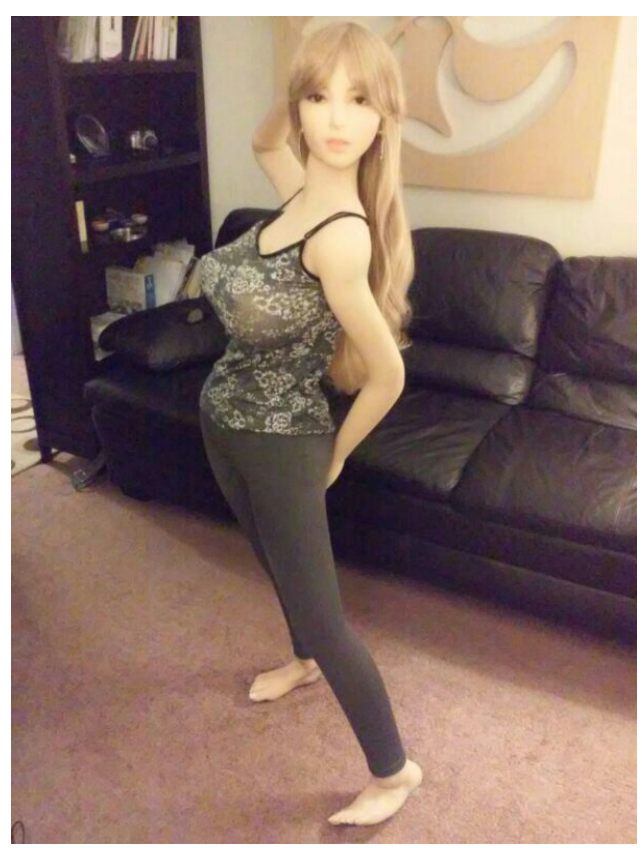

Figure 1: Robot fashion model Victoria Allyn.

*Corresponding author: Allyn JI, RIL Robots, LLC, Los Angeles, USA, Tel: + 3108959109; E-mail: jallyn@rilrobots.com

Received July 14, 2017; Accepted August 04, 2017; Published August 14, 2017

Citation: Allyn JL (2017) How Electricity has Handicapped Robots: Reliance on Electricity has Made Robots Into Rollbots. Adv Robot Autom 6: 174. doi: 10.4172/2168-9695.1000174

Copyright: @ 2017 Allyn JL. This is an open-access article distributed under the terms of the Creative Commons Attribution License, which permits unrestricted use, distribution, and reproduction in any medium, provided the original author and source are credited. 


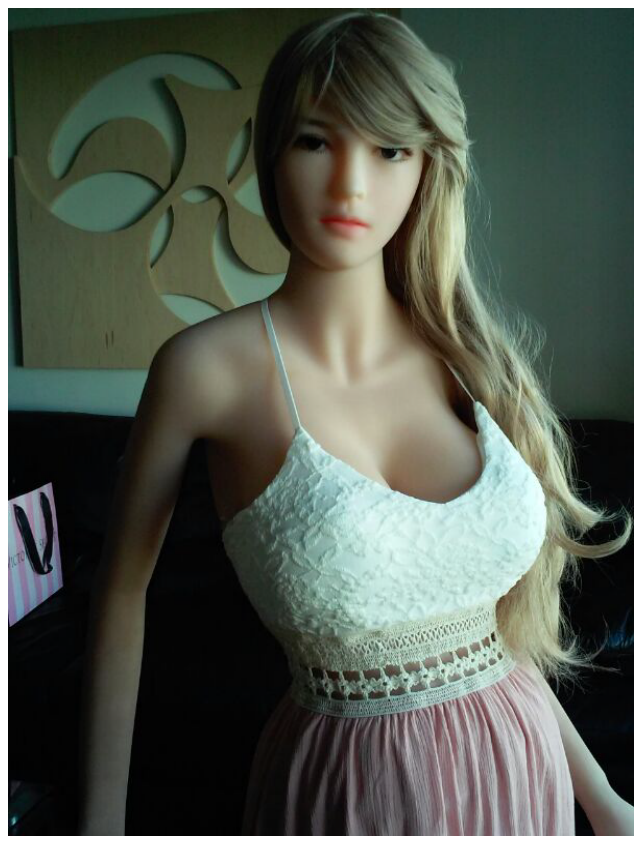

Figure 2: Victoria dress. store mannequins. Not only does the benefit of non-powered walking benefit its endless walking time. But as robot fashion models, they can be dressed in the store's fashions and walked through the store and the mall or shopping center. Showing off the stores fashions and fashion accessories, as if they were fashion models on the runway (Figure 2).

Maybe one day they will even participate in live runway fashion shows. They certainly have the beauty to do so

RIL Robots, LLC may be the first to offer life-size robots that walk without motors or electricity, but it won't be the last. The push for truly green technology will lead other robot makers down the path that RIL Robots, LLC is walking. And who knows. Your next companion you take a moonlight stroll with just might be a RIL Companion from RIL Robots, LLC.

\section{References}

1. RIL Robots (2013) Completely Eliminated Powered Walking From the Locomotion of Its Robots.

2. RIL robot (2013) Robot walking without motors or electricity is even possible. 